\title{
Metódica MEIN para entrenar habilidades metacognitivas en niños de 4 a 6 años. Región Metropolitana de Chile.
}

\section{MEIN Methodical to train metacognitive skills in children from 4 to 6 years old. Santiago Metropolitan Region, Chile}

\author{
Lourdes Ilizástigui del Portal, Thiare Padilla Fernández, Daniela Martinez Sanguinetti y Catalina Fabre López \\ Universidad Santo Tomás, Chile.
}

(Rec: junio de 2019 - Acept: noviembre de 2019)

\begin{abstract}
Resumen
La Metódica MEIN -metacognición infantil- es una herramienta psicoeducativa para educadoras de párvulos y básica, que busca favorecer el desarrollo en las edades de 4 a 6 años, entrenando habilidades cognitivas para desarrollar metacognición en edades más avanzadas. Así, tal estrategia hace posible entrenar de una manera peculiar e interactiva, las habilidades cognitivas frente una actividad, a partir de la propia iniciativa del niño/a. El mediador intenciona, significa y acompaña para que el infante sea capaz de idear la estrategia que empleará en su proceso de aprendizaje, y para que a partir de su apropiación transfiera a otros contextos, valorando el desempeño de su construcción. La metodología empleada fue cualitativa mediante un diseño de estudio de casos. El resultado fundamental del estudio demuestra que la Metódica MEIN es un recurso que facilita a la educadora orientar positivamente los procesos cognitivos en un espacio complejo de desarrollo para el entrenamiento temprano de habilidades metacognitivas. Al niño/a le permite actuar libremente con independencia, facilitando la toma de conciencia.
\end{abstract}

Palabras claves: Entrenamiento, habilidades metacognitivas, estrategia, intermediación.

\begin{abstract}
The MEIN methodical -child metacognition- is a psychoeducational tool for development, aimed at educators to train cognitive skills in children from 4 to 6 years old so that subjects of more advanced ages can develop metacognition. The MEIN methodical makes it possible to train in a peculiar, interactive way, cognitive abilities in the face of an activity that starts from the children's initiative; the mediator promotes, signifies and accompanies them so that they are able to devise the strategy that they will employ in the learning process, and from their appropriation they transfer to other contexts, so as to value their construction's performance. The methodology used was qualitative through a case study design. The fundamental result of the study shows that the MEIN methodical is a resource that facilitates the educator to positively orient cognitive processes in a complex development space for early training of metacognitive skills. The child is free to act independently, facilitating awareness.
\end{abstract}

Keywords: Training, metacognitive skills, strategy, intermediation. 


\section{Introducción}

Los procesos involucrados en el desarrollo de las habilidades cognitivas de la etapa infantil han tenido considerables avances teóricos en su conceptualización. En este sentido, existe un énfasis mayor en la naturaleza social, cultural e interactiva, como también en un rol activo del sujeto, donde la mediación permite intencionar, significar y trascender para favorecer los procesos de la metacognición por medio del entrenamiento temprano de habilidades.

Para abordar la complejidad del desarrollo de habilidades metacognitivas, es necesario y fundamental comenzar a trabajar en su entrenamiento a través de un recurso psicoeducativo que las potencie entre los 4 y 6 años.

Esta misión cobra cada día más importancia, siendo necesario crear métodos que sean capaces de desplegar las potencialidades de los niños/as y que no se limiten solo a la solución de una tarea, sino que avancen más allá, para que puedan ellos o ellas a ser los constructores y controladores de su propia actividad. Esta fue la razón que determinó la creación de la Metódica Metacognición Infantil (MEIN, en adelante), que está orientada a potenciar por medio de un entrenamiento en edades tempranas, las habilidades que favorezcan la metacognición.

La Metódica MEIN, es un intento por investigar y destacar tempranamente la necesidad del entrenamiento de habilidades cognitivas en la cual una educadora crea un espacio de desarrollo para el niño/a y este, mediante la construcción de una actividad a iniciativa de él/ella, diseña una estrategia para obtener un resultado.

El educador/a mediador/a acompaña al niño/a en el proceso de orientación y relacional ante las acciones que realiza para construir una estrategia. Estos procesos permiten entrenar sus habilidades cognitivas y control de su propia actuación, así como de autoevaluar lo realizado.
En este contexto, no se puede negar el esfuerzo que muchos autores han realizado para mejorar los métodos y las técnicas que destacan las potencialidades de los niños/as para alcanzar niveles de desempeño y desarrollo cognitivo, así como para "revelar la condición de sujeto, negada por los enfoques asistencialistas y paternalistas, enfatizar el carácter constructivo del proceso, el carácter interactivo y la libertad metodológica en el desempeño de una tarea" (Ilizástigui, 2014 p.51-53). Sin embargo, el rol activo en la construcción de una actividad a partir de la propia iniciativa, resulta fundamental para desarrollar habilidades que potencien la metacognición.

En este sentido, el entrenamiento cognitivo demuestra que los estudios sobre la metacognición "se interesan sobre todo por el conocimiento de los propios procesos cognitivos y la forma en que influye este conocimiento en los procesos del aprendizaje y su control" (Bustingorry y Jaramillo, 2008). En este orden de ideas, emerge la importancia de la enseñanza de las estrategias cognitivas y metacognitivas, con el fin de proporcionar a los niños/as herramientas necesarias para convertirse en los aprendices autónomos y conscientes de su propio proceso de aprendizaje.

Las estrategias cognitivas se encuentran en el plano del saber hacer con la información, con la actividad y con los elementos del ambiente. El paso a un plano metacognitivo implica la participación de la conciencia como un mecanismo regulador. Esta acción de lo no consciente a lo consciente significa una reconstrucción en el plano de la conceptualización, una transformación de un esquema de acción en un concepto, donde la toma de conciencia no se limita a iluminar aspectos ya dados, sino que construye otros nuevos (Klimenko y Álvarez, 2009).

Siguiendo la misma lógica, se reconoce el aporte realizado por Ilizástigui (2014), quien se encargó de investigar el curso del pensamiento infantil y aporta el Modelo de Intermediación en la Evaluación Cognitiva a través de la Metódica IMEI (Intermediación, Mediación, Exclusión e Inclusión) (Figura 1).

Figura 1

MODELO IMEI (INTERMEDIACIÓN, MEDIACIÓN, EXCLUSIÓN E INCLUSIÓN)
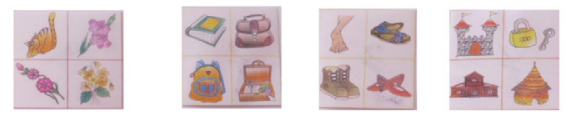

NIVEL I

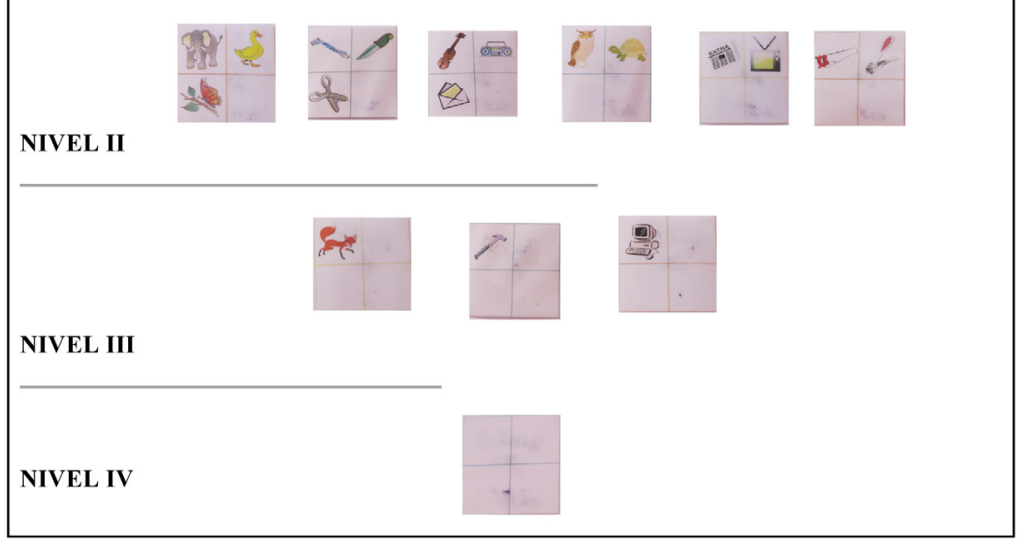

Figura 1. Modelo IMEI (Intermediación, Mediación, Exclusión e Inclusión). Fuente: Ilizástigui, (2014) 
En la Metódica IMEI existe libertad metodológica en la construcción de los objetos de la lámina, realizando los mecanismos del pensamiento de la inclusión/exclusión y otorgándole un significado positivo al error. También se genera una interrelación, donde se le brinda ayuda al niño/a para que logre construir la tarea. El modelo IMEI es un aporte relevante para el desarrollo y orientación del pensamiento, dejando abierta la posibilidad de profundizar en esta dirección, dando paso a desarrollar aspectos potenciadores más que evaluadores en la construcción del sujeto y en la mediación e interacciones entre los actores. De igual forma, busca superar la fase evaluativa, dando paso a una fase desarrolladora y poniendo foco en la elaboración de estrategias para enfrentar una tarea. De esta manera, la creación y diseño de la Metódica MEIN, da un salto cualitativo que apunta a que el niño/a alcance la construcción de la actividad frente a un estímulo con el apoyo entregado por un mediador/a. Por lo tanto, esta Metódica se convierte en una herramienta psicoeducativa.

Este estudio constituye un referente, un recurso que contribuye a enriquecer la línea de investigación asumido por el modelo de intermediación en la evaluación cognitiva de niños/as a través de la Metódica IMEl.

Para esto, es fundamental entender desde un cuerpo teórico lo que propone la Metódica MEIN. El concepto de cognición se refiere a las funciones ejecutivas, tal como han sido estudiadas en la literatura sobre el desarrollo cognitivo, Blair (2013) señala que el concepto orientado hacia la primera infancia, hace referencia a habilidades específicas de procesamiento de información interrelacionadas que permiten y dan paso a: i. la resolución de información conflictiva; ii. la memoria de trabajo, definida como la retención en la mente y la actualización de información al realizar alguna operación en ella; iii. el control inhibitorio, definido como la inhibición de una respuesta predominante o automatizada al dedicarse a la terminación de una tarea; y iv.la flexibilidad mental, definida como la habilidad de cambiar el proceso atencional o cognitivo entre dimensiones o aspectos distintos, pero relacionados en una actividad dada.

La metacognición consiste en dos factores: primero en tomar conciencia de las habilidades, estrategias y recursos que se necesitan para desarrollar una tarea con efectividad, y segundo, en la habilidad para utilizar mecanismos auto-reguladores (como movimientos planificados, evaluación de las actividades en proceso, control del resultado del esfuerzo y solución de dificultades). Una organización funcional del conocimiento (de persona, tarea, estrategia y contexto) y del control (planificación, supervisión y evaluación) de la actividad, es decir, la metacognición, es la comprensión del conocimiento, donde dicho conocimiento es cognición (Flavell citado en Pérez et al. 2017).

En la Metódica MEIN se espera que el niño/a tome conciencia de sus propias acciones y soluciones que realiza ante una actividad. Labarrere (2011) propone una aproximación al despliegue metacognitivo durante la solución de problemas, que debe tener lugar durante todo el proceso, desde su planificación hasta los momentos en los que se producen las acciones de los niños/as respecto de su producto final.

La metacognición es un fenómeno que surge en el crecimiento cognitivo del sujeto como una característica misma del desarrollo de las capacidades mentales. La complejidad de los procesos metacognitivos impulsa a trabajar las habilidades desde la infancia temprana, la que se destaca en la literatura como una etapa importante para el desarrollo del niño/a. Desde esta mirada, Campos (2014) afirma que, en los primeros de años de vida, el cerebro es muy susceptible a las experiencias del ambiente, el cual ejerce gran influencia en las estructuras y circuitos neurales. Por ello, esta etapa es señalada como un periodo sensible, ya que los circuitos neurales son flexibles, plásticos y receptivos a la estimulación ambiental; de esta manera potenciar las habilidades metacognitivas en ese momento de crecimiento neuronal y sensible para el desarrollo de destrezas básicas puede tener efectos duraderos para aprender y almacenar información.

Hassuinger et al (2017) señala que el juego desempeña un papel importante en la infancia, otorgando herramientas efectivas para apoyar los objetivos de aprendizaje. Tal actividad implica diversión, sentido de la curiosidad, tomar una actitud participativa, generar significados y ser socialmente interactivos. Por esto, introducir al niño/a a través de la estimulación con juegos lúdicos favorece al procesamiento de la información, apuntando a que el ser humano es capaz de manipular información simbólica. Para efectos de este estudio, nos situamos desde la etapa preoperacional que se extiende de los 2 a 7 años, caracterizándose por el uso de símbolos y representaciones sin tener contacto con los objetos directamente para evocar o pensar en un objeto, persona o evento (Piaget, 1933). De esta manera el uso de símbolos es fundamental para el entrenamiento metacognitivo en cuanto al proceso de construcción de la actividad.

Por otra parte, desde la teoría sociocultural se consideran los fundamentos de Vygotsky (1985,1982), el cual enfatiza la participación de los sujetos con su ambiente y contempla el desarrollo cognoscitivo como parte de un proceso colaborativo. Afirma que los sujetos aprenden por medio de una interacción social, adquieren habilidades cognitivas como parte de una inducción a una forma de vida. Desde esta perspectiva: "(...) todas las funciones en el desarrollo del niño aparecen en dos momentos: primero a nivel social, y después a nivel individual; en primer lugar, entre las personas (inter-psicológicamente) y después en el interior del propio niño (intra-psicológicamente) (Vygotsky, 1968). A raíz de esto, es posible insertar otra tesis importante de este autor, que plantea la Zona de Desarrollo Próximo ( $Z D P)$, consistente en "la distancia entre el nivel actual de desarrollo, determinado por la capacidad de resolver independientemente un problema y el nivel de desarrollo potencial, determinado a través de la resolución de un problema bajo la guía de un adulto o en colaboración con un compañero más capaz" (Vygotsky, 1979, p.133).

En este sentido, para que el entrenamiento temprano de habilidades metacognitivas exista, es fundamental un mediador/a que sea capaz de guiar y acompañar al niño/a, con el propósito de que este sea capaz de adquirir un nuevo conocimiento conscientemente para que posteriormente, pueda transferirlo a otros contextos de su vida. Este mediador/a ocupa un rol de andamiaje, concepto utilizado como una metáfora para representar el proceso de aprendizaje, pues supone un apoyo temporal que se proporciona a un niño/a para hacer una actividad hasta que pueda hacerla por sí mismo (Vygotsky, 1979). 
En cuanto a la dimensión interrelación, el autor nos plantea que la unidad del análisis del proceso de enseñanza-aprendizaje ya no es la actividad individual del estudiante, sino la actividad articulada y conjunta. La actividad del niño/a que está a la base del proceso de construcción del conocimiento se inscribe en el marco de la interacción conjunta (Coll, 1997). Resulta de vital importancia, porque el sujeto no realiza la construcción y ejecución de una actividad de forma aislada y unidireccional, sino que es una construcción colaborativa y mediada entre dos personas, y es a partir de esa vivencia que se genera una interrelación entre el niño/a y el mediador/a, quien acompaña y guía al sujeto en la experiencia de aprendizaje.

Asimismo, como plantea Labarrere (2011), la interacción guiada con el problema debe ser llevada hasta un punto en que el niño/a pueda extraer, generalizar y transferir las implicaciones personales frente a la actividad, de tal forma que permita su desarrollo cognitivo, y donde interactúe con la Metódica a través de la mediación que se va generando con la persona adulta.

Siguiendo la misma línea sobre la construcción colaborativa entre mediador/a y niño/a, Feuerstein (1986), plantea el concepto de la experiencia de aprendizaje mediado (EAM), donde el mediador motivado a intervenir con el niño/a, intenciona, trasciende y da significado al aprendizaje permitiendo generar modificación en la cognición (Molina y Fondos, 1996).

Muchos autores han centrado sus esfuerzos en la creación y mejoramiento de los métodos y técnicas que, desde las conceptualizaciones teóricas y metodológicas, contribuyen a que el mediador potencie los procesos cognitivos. Este estudio se centró en el carácter de agente activo del sujeto, es decir que, a través de la Metódica MEIN, él o ella construya su actividad y estrategia apoyado por quien medie, posibilitando el entrenamiento de las habilidades metacognitivas tempranamente.

La Metódica MEIN intenta que los cuadernillos destinados a profesionales de la educación para el entrenamiento del desarrollo de habilidades metacognitivas de los niños se alejen de las lógicas normativas y mecanizadas, dando paso a variables más auténticas que evidencien la construcción de una estrategia por sobre la medición, a partir de la ayuda de un mediador/a y la interacción entre los actores, promoviendo con ello que el sujeto sea activo frente a una actividad. Por lo tanto se espera dar un salto cualitativamente importante de lo instrumental a lo desarrollador.

En esta interacción de reciprocidades, significados, trascendencia e interrelación, se pretende que el niño/a sea un actor consciente de su propio conocimiento. Que aprenda temprana y progresivamente a construir en la relación con un mediador/a la estrategia para construir la actividad deseada.

En este sentido, la Metódica MEIN releva la condición de sujeto, enfatizando el proceso y construcción de conocimiento de quienes deben interactuar para lograr entrenar las habilidades orientadas al desarrollo metacognitivo. Se reconoce el potencial cognitivo a partir de su propia construcción, el niño/a goza de una libertad metodológica donde se evidencian las diferentes formas y maneras de resolver un problema, se intenciona y estimula a que el niño explique sus acciones de cómo llega a su producto final, esto con la ayuda brinda- da por el/la mediador/a, produciendo espacios de interacción entre ambos. Esto permite expresar el desarrollo o proceso de su construcción, cómo va regulando sus procesos cognitivos y aprendizajes, tomando en consideración el uso de conocimientos previamente aprendidos y/o asociación de elementos. A partir de esto, las investigadoras de este estudio plantean la siguiente pregunta para responder:

- ¿Cómo se manifiesta la complejidad del espacio de intermediación (Adulto- Niño/a) en la aplicación de la Metódica MEIN como estrategia orientada al desarrollo y entrenamiento temprano de habilidades metacognitivas?

La creación y diseño de la Metódica MEIN, orienta a favorecer el entrenamiento metacognitivo de niños entre 4-6 años de edad, además constituye un intento por develar en profundidad la compleja trama de acciones y control de las mismas, así como orientaciones que tendrán lugar durante la aplicación por parte del mediador/a, constituyendo una herramienta potente para entrenar la metacognición en plena etapa de desarrollo infantil. Se centra en que el infante es un agente activo en desarrollo y arrojará luz en torno a su desempeño frente a la construcción y proceso de la actividad que deberá construir. Es, además, una manera de entender la mediación como vía de formación y entrenamiento de la metacognición, donde el producto depende de ambos: sujeto y mediador tratando de insertar la interacción entre ellos de manera conjunta.

Por tanto, la Metódica MEIN tiene por objetivo: Objetivo General

- Caracterizar y analizar las complejidades de espacio de desarrollo metacognitivo en la Metódica MEIN.

\section{Método}

Este estudio es una investigación cualitativa (Iñiguez, 1999), que permitió constatar las complejidades del proceso y estrategias que se fueron configurando en la aplicación de la Metódica MEIN, siendo de tipo inductivo, ya que el análisis fue desde la acción y construcción de lo que relataban los participantes, especialmente los infantes. Esto permitió evidenciar el despliegue de las habilidades y estrategias en un espacio desarrollador, donde el mediador sin interferir en lo que niño/a decía o hacía, colaboró con la finalidad de entrenar las habilidades metacognitivas. De esta manera, la investigación está orientada a comprender e interpretar las acciones, diálogos y los significados de los niños/as que formaron parte de la muestra, con la finalidad de obtener en su medio natural, todo lo concerniente al proceso de entrenamiento de las habilidades metacognitivas (Mella, 1998). Para el diseño de investigación, se consideró el estudio de casos (Piñuel, 2002), permitiendo un análisis detallado de la situación de cada niño/a al momento de la aplicación, buscando lo particular de cada uno, dado que cada sujeto presentó diferentes resultados en la Metódica MEIN.

\section{Participantes}

En este estudio participaron 6 niños/as, 2 niños/as de cada edad, 4, 5 y 6 años pertenecientes a la Región Metropolitana de Chile. 
Criterios de inclusión: los niños/as se encontraban en el rango de edad mencionado, sin estar diagnosticados por especialistas con trastornos del desarrollo o en riesgo de padecerlos.

Por otra parte, los criterios de exclusión fueron: niños/as mayores de 6 años o menores de 4 y que estuvieran diagnosticados por especialistas con trastornos del desarrollo o en riesgo de padecerlos.

\section{Técnica de Producción de Datos}

Metódica MEIN. Es una guía de trabajo que cuenta con 20 láminas que componen el cuadernillo, agrupadas por edad desde los 4 a 6 años. Las seis actividades correspondientes a la edad están ordenadas con el mecanismo de "dificultad creciente" es decir, estratégicamente de menor a mayor complejidad, para entrenar el esfuerzo cognitivo y construcción de estrategias por parte del niño/a. Se aplica de forma individual.

En las actividades que construye el niño/a en la Metódica MEIN, se estimulan las habilidades por áreas, apuntando a las dimensiones importantes de la primera infancia, como discriminación perceptiva, desarrollo cognitivo, lenguaje y comunicación, psicomotricidad, socialización y autoayuda, per- mitiendo entrenar las habilidades metacognitivas a través de la construcción de una actividad a partir de la iniciativa, desempeño del niño/a y solución de los problemas presentados en el cuadernillo de trabajo. Por medio de este se entrenan habilidades y destrezas, donde el carácter lúdico permite el desarrollo social, fomentando la cooperación y la afectividad a partir de los vínculos establecidos entre los actores involucrados. En este sentido, el mediador/a y el niño/a crean un espacio de desarrollo e interrelación de aprendizajes mutuos y compartidos, situándose desde un vértice fundamental para generar nuevos aprendizajes y diálogos.

El eje central es ir entrenando mediante preguntas abiertas las acciones que le permiten al niño/a ir construyendo la estrategia para enfrentar la actividad. Se busca la construcción espontánea y libre del niño/a frente al estímulo, se dispone de todo el tiempo necesario y no hay puntos de valores (buenas o malas) sino más bien es una actividad que está orientada al proceso propiamente tal y no a la solución del resultado. La consigna es: "Mira esta lámina, debes observarla con atención, necesitamos que construyas algo con ella. Puedes preguntar las veces que desees, te ayudaremos" (Figura 2).

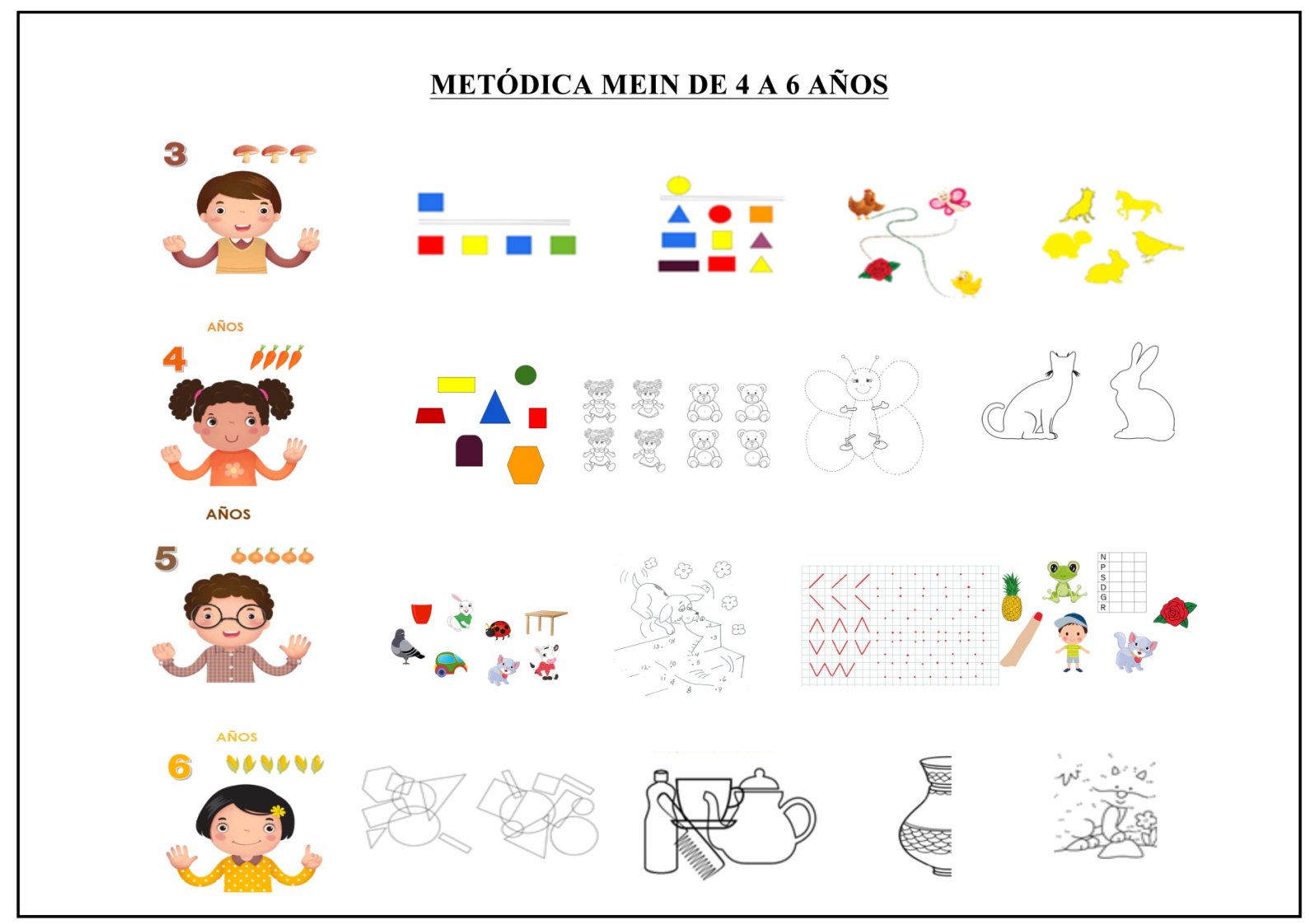

Figura 2. Metódica MEIN de 4 a 6 años. Fuente: Elaboración propia. 
La Metódica MEIN, está diseñada por 3 fases: i. Familiarización, ii. Intermediación entre adulto y niño(a), y iii. Autoevaluación.

Fase l: Familiarización.

Corresponde y se caracteriza por corroborar la orientación del niño frente al espacio de desarrollo que el mediador prepara con anterioridad, donde el elemento movilizador es el juego, siendo el medio de vinculación y comunicación entre los actores, y buscando la familiarización con el estímulo que se muestra en este espacio. En esta fase inicial, al niño/a se le presentan dos láminas de fácil construcción, correspondiente a la edad anterior del niño, por ejemplo al niño/a de 4 años se parte con las dos últimas láminas de 3 años. En este momento el protagonista ya está construyendo su actividad, y se le plantean preguntas abiertas para favorecer en entrenamiento como: ¿qué puedes hacer con esto?, ¿te imaginas algo?, ¿puedes relacionarlo con algo que conociste antes?, ¿qué ideas tienes?, según las necesidades de niño/a. Frente a la actividad, la dimensión orientación establece qué hace o dice el niño/a cuando trabaja e interactúa con el estímulo (lámina): si pregunta para orientarse, si se esfuerza para realizar la actividad, si se fatiga o si está desmotivado y requiere estimulación, si es de acercamiento directo, distante o muestra una estrategia para construir el producto final. Se debe generar un espacio de confianza y seguridad para el niño/a.

Fase II: Intermediación entre adulto y niño/a.

Corresponde la interacción que se genera entre los actores. Se le presentan al niño/a cuatro láminas según su edad, una a una, a partir de las cuales deberá construir la actividad. En este momento, el mediador intenciona preguntas relacionadas con la construcción de esta, como, por ejemplo: ¿dime cómo lo hiciste?, ¿qué harás primero?, entre otras. El adulto mediador/a favorece la construcción a través de preguntas y orientaciones con el propósito de entrenar las habilidades, acciones, estrategias que emplea el niño/a para generar la toma de conciencia de lo que ejecuta y así lograr trascender lo aprendido a las siguientes láminas. El niño/a construye la actividad con la mediación, pero con libertad metodológica ante el estímulo. Aparecen las diversas formas de expresar una respuesta elaborada por el niño/a por medio de: estrategias, acciones, evocación de aprendizajes previos y asociación de elementos. Tiene como propósito poner en manifiesto todos los recursos del niño para construir su producto final. La condición de sujeto es revelada como activa, se despliega su desarrollo cognitivo y regulación de su actuación. Otro indicador importante es el tipo de relación que se genera entre ambos para enfrentar la construcción de la actividad: si pide orientaciones, ayudas, aclaraciones. Es importante también la conducta del niño, en tanto si es valorada de forma desconcentrada, impulsiva o intranquila.

Fase III: Autoevaluación.

La autoevaluación cobra un sentido importante, donde el niño/a, a través de su propia valoración, evalúa su actividad y desempeño de su producto final. Por su parte, el mediador pregunta: ¿cómo crees que lo hiciste?, ¿te esforzaste para hacer la tarea?, ¿te resultó fácil realizar la tarea?, ¿por qué?, ¿te gustó como te quedó? Esta dimensión es relevante para el proceso de toma de conciencia que desarrolla el niño/a, siendo crítico sobre su actuar.

Otro instrumento aplicado fue la observación no participante
(Kawulich, 2005), la cual resultó fundamental para vislumbrar, percibir y evidenciar todas aquellas acciones que realizó el niño/a al momento que se aplicó la Metódica MEIN, donde había que tomar especial atención en cómo el niño/a ejecutaba la actividad que era construida por él/ella, observando su comportamiento, lenguaje y estrategias que emplearon para la construcción de la actividad.

Finalmente, el apoyo de video. En virtud de que durante el proceso de aplicación no se perdiera ningún aspecto importante para el estudio y posterior análisis de los resultados, resultó fundamental utilizar el apoyo de video en la aplicación para abordar con mayor precisión la información obtenida durante el proceso de entrenamiento metacognitivo.

Procedimiento de producción de información

En este estudio se consideró relevante analizar las acciones, diálogos que surgieron a través de la aplicación, además de la conducta y las ayudas solicitadas. La técnica utilizada fue el análisis de contenido, definido como un conjunto de procedimientos interpretativos que son previamente registrados de procesos singulares y que tiene como objetivo elaborar y procesar los datos relevantes del contenido, generando una combinación de categorías (Piñuel, 2002).

Dada la cantidad de información que se obtuvo, fue necesario y pertinente identificar aquellas unidades de análisis más relevantes y subunidades relacionadas con la acción, discurso y conducta demostrada para realizar la actividad por parte de los participantes en el estudio y análisis posterior. En virtud de la necesaria articulación, el diseño de esta investigación consideró los planos A (orientación), B (relacional), C (conductual) y $D$ (evaluación de la actividad) para facilitar la interpretación y comprensión del proceso de entrenamiento de las habilidades metacognitivas.

A continuación, se expone los niveles que se realizaron para hacer un análisis detallado:

Primer nivel de análisis. Aplicación, transcripción y análisis de la Metódica MEIN, además de analizar los videos (por cada niño/a participante).

Segundo nivel de análisis. Descripción de las acciones realizadas por cada niño/a.

Categoría Orientación: establece cómo trabaja el niño/a con el contenido de la lámina, tomando atención en qué dice o qué hace, si es de ejecución directa e inmediata o si muestra alguna estrategia frente a la resolución del problema, si construye la actividad y/o si solicita ayuda. Muestra cómo genera la "orientación", es decir, si logra ubicar los objetos presentados en las láminas para la construcción de la actividad.

Categoría Relacional: establece la interacción con el adulto mediador a través de preguntas, solicitud de ayuda, aclaraciones o si busca apoyo. Otro aspecto puede ser que el niño/a, no solicite ayuda para construir la actividad, si construye la relación con el mediador/a o no.

Categoría Conductual: establece la actitud del niño/a frente a la solicitud de la construcción de la actividad. Puede mostrar una conducta intranquila, desconcentrado, impulsivo, falta de motivación, fatigado, entre otras. 
Categoría Autoevaluación: establece la evaluación de la actividad construida por el niño/a, si le gustó o no, cómo le resultó o si le quedó bien o mal desde su autoanálisis.

Tercer nivel de análisis. Análisis integrativo por cada niño/a, considerando los cuatro planos de acción mencionados anteriormente.

\section{Resguardos Éticos}

Se consideró el consentimiento informado para los padres de los participantes, donde se explica la finalidad del estudio y se autoriza la participación en él. También se destaca la formalidad, responsabilidad y puntualidad por parte de las investigadoras. $Y$ por otro lado desde el código de ética (1996) el Artículo №15 (Aspectos relativos a la investigación en Psicología). Finamente, se hizo la devolución de manera escrita y oral sobre los resultados obtenidos en la investigación a los padres de los niños e institución que participó. Se resguardaron todos los nombres, tanto de los participantes como de la institución que participó.

\section{Resultados}

En consideración a la cantidad de información que se obtuvo, a continuación se describe y analiza en detalle, un caso de 6 años que es representativo del proceso llevado por el resto de los participantes.

Los resultados consisten en el análisis de las acciones y procesos de construcción frente a los estímulos propuestos de la Metódica MEIN. En primer lugar, aparecen los procesos que generaron los participantes en las 3 fases diseñadas. Además, se describen los planos de orientación, relacional, conductual y autoevaluación, así como las acciones e interrelaciones entre ambos actores frente a la solución de problema, analizando tanto el rol del protagonista como el del adulto mediador. En segundo lugar, se presenta un análisis integral cualitativo que da cuenta del proceso del niño/a frente a la actividad.

Tabla 1: Procesos y operaciones de entrenamiento metacognitivo a través de la Metódica MEIN

\begin{tabular}{c} 
METODICA MEIN \\
\hline FASE
\end{tabular} $\begin{gathered}\text { MLDIADOR } \\
\text { Mencionó la consigna al inicio de }\end{gathered}$




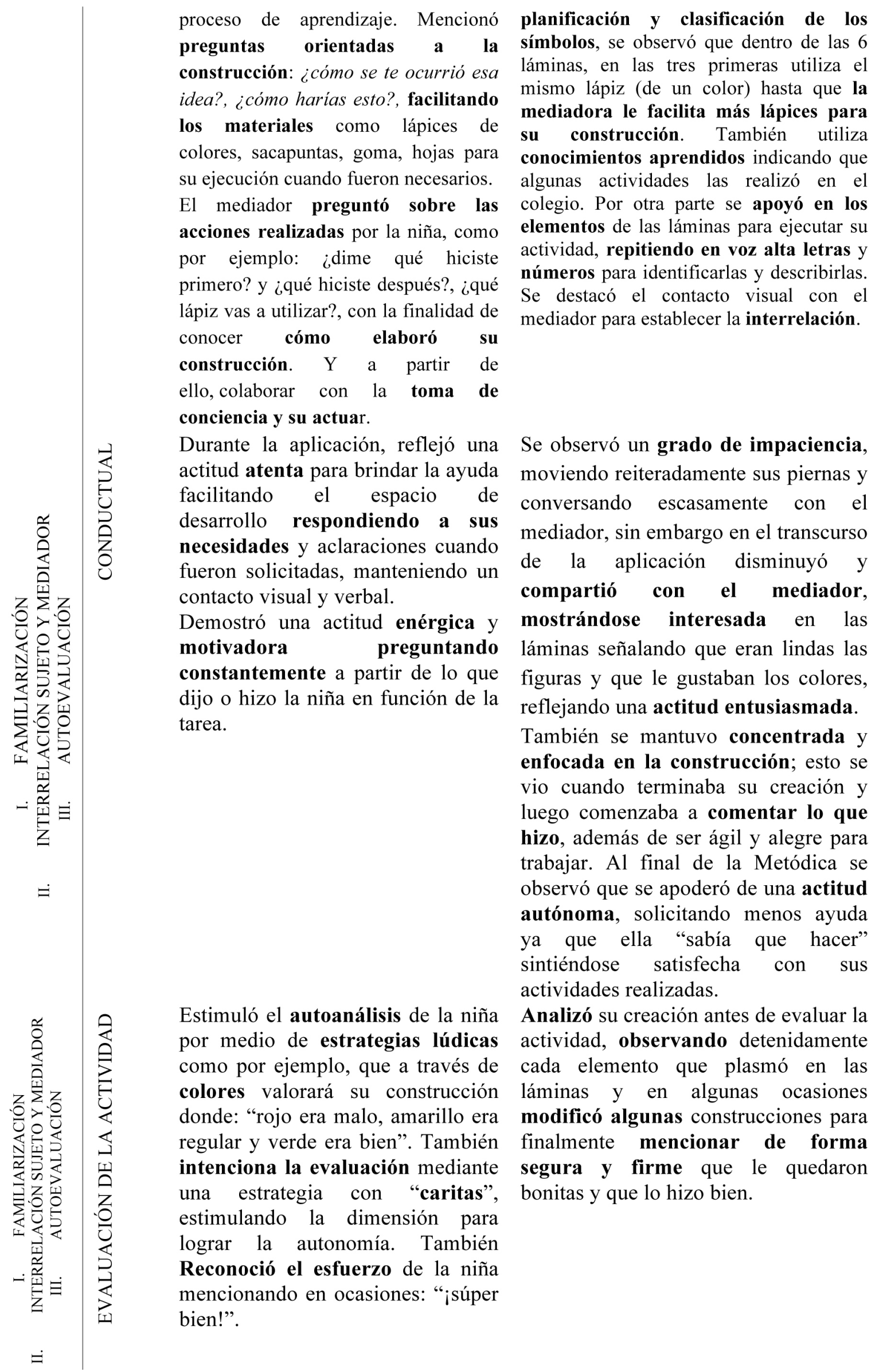

Nota. Elaboración propia de análisis de información a partir de la aplicación Metódica MEIN 


\section{Análisis integral de Niña $B$}

En cuanto al plano de orientación frente a la actividad, se observa en el protocolo que la niña se orientó adecuadamente observando detenidamente e interactuando con las láminas. Como principal estrategia, se destaca la observación al momento de su construcción, además del uso de conocimientos previos aprendidos en casa y colegio, los cuales le permitieron planificar y organizar sus pasos para la ejecución de su producto final pudiendo verbalizar sus actos. En este proceso el mediador la acompañó e intervino con preguntas que la ayudaron a su construcción.

En cuanto al plano relacional, se pudo observar hubo mayor interacción con el mediador, pidiendo orientaciones y ayudas para realizar las actividades. Por otro lado, la acción potenciadora, se da brindándole ayuda en algunas palabras o letras que le dificultaron; esto retroalimentó la realización de las actividades de manera conjunta. El mediador intencionó y acompañó constantemente la construcción de la actividad mediante preguntas orientadoras. La niña solicitó ayuda asistida durante el transcurso de la aplicación, buscando apoyo a través del mediador, por tanto, se puede constatar que se construye la relación con el mediador a partir de los contenidos de las láminas.

En cuanto al plano conductual, se mantuvo tranquila y concentrada, mostrando motivación y deseos de realizar las actividades de forma pausada y calmada. Se generó una interacción colaborativa que facilitó su proceso de aprendizaje. Al final de la aplicación se empodera de su actitud y se muestra independiente y autónoma, requiriendo de menos mediación. En cuanto al plano de evaluación, autoevaluó su construcción como fácil, mencionando que se esforzó por realizar la tarea, y que le gustaron las construcciones que realizó en las láminas de la Metódica.

\section{Discusión}

La Metódica MEIN es un recurso psicoeducativo para el desarrollo de habilidades metacognitivas en niños/as y para el desempeño del docente en un rol de mediador, donde se genera un proceso interactivo en el cual el adulto acompaña al infante por medio de la mediación. Este ejercicio, se constituye como un recurso didáctico que hace posible entrenar al niño/a de una manera peculiar e interactiva en la realización de una actividad, donde durante su aplicación el niño/a interactúa como si fuera parte de un juego. Si no hay un entrenamiento previo de las habilidades y del rol mediador empleado, no tiene suficiente impacto en los proceso metacognitivos futuros.

Este recurso no es un instrumento evaluativo donde se le dice qué hacer al niño/a, sino que se le da importancia a generar una relación gratificante previa por medio de la familiarización y sensibilización para avanzar hacia a un plano desarrollador. Se requiere de mayor estimulación, intencionalidad y acompañamiento para facilitar el proceso de aprendizaje de los niños/ as, que los lleven a idear una estrategia para la construcción solicitada. A pesar de esto, se observa que las orientaciones dadas a los niños/as se orientan y conducen al resultado por sobre el proceso de construcción de las actividades, donde se despliegan las acciones para enfrentarse a la resolución de estas, existiendo una tendencia a resolver de manera mecanizada, sin análisis profundos sobre el proceso de mediación entre los actores.
Esto nos hace pensar que la mediación contribuye como un eje central en el acompañamiento del niño en su proceso de construcción y desarrollo cognitivo. Es por esto que se requiere de un rol mediador que enseñe, guíe y acompañe en la construcción y, en este sentido, la formación de profesionales cobra importancia para emplear con efectividad este rol para alcanzar la trascendencia y significancia de los procesos metacognitivos posteriores. Existe una necesidad de brindar una interacción de calidad por parte del mediador, considerando el complejo espacio que se genera entre los actores. Debe tener lugar la intencionalidad directa y explícita por parte del educador mediador, para alcanzar mayor autonomía y trascendencia de los aprendizajes desplegados en la ejecución del producto final.

Se revela la necesidad de seguir trabajando en orientaciones que potencien las habilidades metacognitivas desde edades tempranas. Resulta relevante que existan instancias en las escuelas y jardines orientadas al trabajo de habilidades metacognitivas, donde se potencie el rol activo del sujeto y la capacidad de apropiarse de la actividad dada junto con el acto de mediación, incorporando como estrategia potenciadora la metacognición en la interacción niño-mediador.

En relación con el diseño de investigación, es importante señalar que el uso de estudios de casos resultó fundamental y apropiado para la metodología que fundamenta la investigación. A nivel de las categorías de análisis, se pudo apreciar aspectos positivos en el desarrollo del entrenamiento de las habilidades metacognitivas. En el plano orientación, en general, los participantes muestran una buena capacidad para entender y orientarse a realizar su producto, mostrándose concentrados y focalizados en su actividad. En el aspecto relacional, a pesar de no pedir o solicitar ayuda de manera constante y continua, los participantes integran al mediador como parte de su construcción, buscando una vinculación y acompañamiento en el proceso.

En cuanto a la esfera de autoevaluación, los participantes son agudos al momento de describir y evaluar su actividad. Además, los participantes desplegaron maneras creativas para resolver sus actividades, recurriendo al uso de aprendizajes previos y agudeza en su narrativa ante la solución de su tarea

Como punto de análisis crítico y limitaciones frente a la investigación, las autoras de este estudio consideraron que el rol empleado fue desarrollado de forma escasa y débil, dando cuenta la dificultad de asumir el "rol mediador" que exige la Metódica en su aplicación y acompañamiento con el niño/a.

En base a una profunda reflexión, la explicación en cuanto al rol desempeñado tiene relación con la necesidad de entrenar, más aún el modo de enfrentarse al espacio de intermediación para entrenar, intencionar y destacar las potencialidades y recursos de los niños frente a una actividad. A pesar de que existe detrás del mediador una enseñanza que estimula un rol activo en el campo evaluativo, es importante integrar ambas miradas que permitirán llegar a una visión integral del sujeto, mirando aspectos evaluativos y potenciadores. El estudiante tiene que apropiarse de un rol activo para traspasar, entender e integrar ambas capacidades importantes a la hora de mirar a un sujeto, especialmente en el espacio brindado por la Metódica MEIN, que busca un salto cualitativo y desarrollador en los niños/as. Por dicho motivo, resulta fundamental seguir 
formando profesionales en el área de psicología orientado a potenciar el rol activo del estudiante, donde se continúe potenciando aún más las habilidades y competencias necesarias para asumir rol mediador.

Es por esto que a partir de la experiencia de esta investigación, se concluye que resulta fundamental seguir transitando desde el modelo paradigmático y concentrar los esfuerzos de formación en entrenar y potenciar el rol mediador. Esto debe ser previo a la potenciación metacognitiva, ya que es la manera y vía efectiva para colaborar y acompañar al niño en el desarrollo de habilidades y estrategias metacognitivas para la resolución de una actividad.

Por otra parte, como limitación de esta investigación, resultaría interesante para poder abordar con mayor precisión el fenómeno de la metacognición a través de la Metódica MEIN, considerar un mayor número de participantes, diversos contextos y necesidades de los niños/as que den cuenta de una diferencias significativas y enriquecedoras de los resultados que se pudiesen obtener.

En cuanto a las perspectivas futuras, la intenciones de la investigación es continuar este camino iniciado, con el propósito de contribuir al desarrollo y aprendizaje de los niños entre 4 a 6 años, y por otro lado, seguir enriqueciendo la Metódica MEIN con la intención de que sea una guía que se pueda implementar de manera grupal, buscando que entre los mismo actores puedan entrenarse mutuamente para el desarrollo cognitivo y ser una herramienta psicoeducativa con modalidad grupal para favorecer el despliegue de habilidades que permitan dirigir y medir las acciones entre ellos mismos.

\section{Referencias}

Bustingorry, S. y Jaramillo, S. (2008). Metacognición: Un camino para aprender a aprender. Estudios pedagógicos (Valdivia), 187-197.

Blair, C. (2013). Funciones Ejecutivas en el salón de clases. Enciclopedia sobre el desarrollo de la primera infancia. 1-6, Recuperado de http://www.enciclopediainfantes.com/sites/default/files/textes-experts/ es/2480/funciones-ejecutivas-en-el-salon-declase.pdf

Campos, A. (2014). Los aportes de la neurociencia a la atención y educación de la primera infancia. Bolivia: Cerebrum.

Coll, C. (1997). Aprendizaje escolar y construcción del conocimiento. España: Editorial Paidós.

Código de Ética del Colegio de Psicólogos de Chile (1996)

Feuerstein, R. (1986). Experiencia del aprendizaje mediado. Siglo Cero. 106, 28-32

Hassuinger- Das,B. Toub, T. Zosh, J, Michnic. Golinkoff, R. \& Hirsh- Pasek (2017) More than just fun: a place for games in playful learning / Más que diversión: el lugar de los juegos reglados en el aprendizaje lúdico. Recuperado de https://www.tandfonline.com/doi/ full/10.1080/02103702.2017.1292684?scroll=top\&n eedAccess $=$ true

Ilizástigui, L. (2014). El modelo de intermediación en la evaluación cognitiva de niños. Madrid: Dykinson.

Iñiguez, L. (1999). Investigación y evaluación cualitativa: bases teóricas y conceptuales. Revista Atención Primaria,
23(8), 108-122.

Kawulich, B. B (2005). La observación participante como método de recolección de datos. Forum: Qualitative social research, 6,1-32.

Klimenko,O. y Álvarez,J. (2009). Aprender cómo aprendo: La enseñanza de estrategias metacognitivas. Educación y educadores, 12(2), 11-28.

Labarrere, A. (2011). Funcionamiento cognitivo y desarrollo en ZDP. Pensamiento Educativo. Revista de Investigación Educacional Latinoamericana, 32(1), 141-154.

Mella, O (1998). Naturaleza y Orientaciones Teórico-Metodológicas de la investigación Cualitativa. Recuperado de http://www.reduc.cl/wp-content/ uploads/2014/08/ Naturaleza-y-orientaciones.pdf

Molina,S. y Fondos, M. (1996) Educación cognitiva I. Zaragoza: Mirá.

Piaget, J (1933). La representación del mundo en el niño. Madrid: Morata

Pérez Clarice, D. y Cardelle, F. (2017) Cuestionario PIE-TDA-H. Programa de intervención educativa de primaria en estrategias cognitivas y metacognitivas para niños con dificultades de aprendizaje - TDA-H. Revista Electrónica de terapia ocupacional Galicia. TOG, 14. 325-339

Piñuel, S. (2002) Epistemología. Metodología y técnicas del análisis de contenido. Estudio de sociolinguística. 3(1), 1-42.

Vygotsky, L. (1979): El desarrollo de los procesos psicológicos superiores. Buenos Aires: Grijalbo.

Vygostsky, L. (1985,1982). Obras completas, Tomo I,II Y IV. Visón.

Vygostky, L (1968). El desarrollo de los procesos psicológicos superiores. Barcelona: Critica. 